\title{
Effect of Modafinil on Learning and Task-Related Brain Activity in Methamphetamine-Dependent and Healthy Individuals
}

\author{
Dara G Ghahremani*,', Golnaz Tabibnia ${ }^{2}$, John Monterosso ${ }^{3}$, Gerhard Hellemann', Russell A Poldrack ${ }^{4}$ \\ and Edythe D London*, $1,5,6$ \\ 'Department of Psychiatry and Biobehavioral Sciences, University of California, Los Angeles, CA, USA; ${ }^{2}$ Department of Social and Decision \\ Sciences, Carnegie Mellon University, Pittsburgh, PA, USA; ${ }^{3}$ Department of Psychology, University of Southern California, Los Angeles, CA, USA; \\ ${ }^{4}$ Departments of Psychology and Neurobiology, University of Texas, Austin, TX, USA; ${ }^{5}$ Brain Research Institute, University of California, \\ Los Angeles, CA, USA; 'Department of Molecular and Medical Pharmacology, University of California, Los Angeles, CA, USA
}

Methamphetamine (MA)-dependent individuals exhibit deficits in cognition and prefrontal cortical function. Therefore, medications that improve cognition in these subjects may improve the success of therapy for their addiction, especially when cognitive behavioral therapies are used. Modafinil has been shown to improve cognitive performance in neuropsychiatric patients and healthy volunteers. We therefore conducted a randomized, double-blind, placebo-controlled, cross-over study, using functional magnetic resonance imaging, to examine the effects of modafinil on learning and neural activity related to cognitive function in abstinent, MA-dependent, and healthy control participants. Modafinil (200 mg) and placebo were administered orally (one single dose each), in counterbalanced fashion, $2 \mathrm{~h}$ before each of two testing sessions. Under placebo conditions, MA-dependent participants showed worse learning performance than control participants. Modafinil boosted learning in MA-dependent participants, bringing them to the same performance level as control subjects; the control group did not show changes in performance with modafinil. After controlling for performance differences, MAdependent participants showed a greater effect of modafinil on brain activation in bilateral insula/ventrolateral prefrontal cortex and anterior cingulate cortices than control participants. The findings suggest that modafinil improves learning in MA-dependent participants, possibly by enhancing neural function in regions important for learning and cognitive control. These results suggest that modafinil may be a suitable pharmacological adjunct for enhancing the efficiency of cognitive-based therapies for MA dependence.

Neuropsychopharmacology (20I I) 36, 950-959; doi:I 0. I038/npp.20 I0.233; published online 2 February 201 I

Keywords: modafinil; fMRl; methamphetamine; anterior cingulate; learning; drug abuse

\section{INTRODUCTION}

Methamphetamine (MA) abuse and dependence are significant public health problems both in the United States (Gonzales et al, 2010) and worldwide (Pluddemann et al, 2010; Rawson and Condon, 2007; United Nations Office on Drugs and Crime, 2009; Wechsberg et al, 2010). Behavioral treatments, such as cognitive behavioral therapy, are the principal therapeutic strategy used for MA dependence, but they have only achieved modest success (Lee and Rawson, 2008). One reason for the limited efficacy of such therapeutic interventions may be the cognitive impairments associated with chronic MA abuse (eg, London et al, 2005; Monterosso et al, 2005; Salo et al, 2005; Scott et al, 2007;

* Correspondence: Dr DG Ghahremani or Dr ED London, Department of Psychiatry and Biobehavioral Sciences, UCLA Semel Institute, 740 Westwood Plaza, C8-83I, Los Angeles, CA 90095, USA, Tel: + I (310) 825 0606; Fax: + I (310) 825 08।2, E-mail: darag@ucla.edu or elondon@mednet.ucla.edu

Received 17 September 20 I0; revised 5 November 20 I0; accepted 17 November 2010
Simon et al, 2010). These impairments may interfere with behavioral treatment, leading to poor treatment retention and outcome (Vocci, 2008). To improve treatment outcomes, cognitive remediation strategies using pharmacotherapy have been proposed, and several psychotropic medications have been identified as potential candidates (Sofuoglu, 2010; Vocci, 2008).

Modafinil (2-[(diphenylmethyl) sulfinyl] acetamide) is an analeptic drug, approved for the treatment of narcolepsy and used primarily to promote wakefulness (Wesensten et al, 2002), but it has gained increasing popularity as a cognition-enhancing agent in patients and also in healthy individuals (Greely et al, 2008; Sahakian and Morein-Zamir, 2007; Stix, 2009). It has been shown to improve cognitive performance in healthy research volunteers (Randall et al, 2005; Repantis et al, 2010; Turner et al, 2003), with a larger effect in patients with neuropsychiatric disorders such as attention-deficit hyperactivity disorder and schizophrenia (Turner et al, 2004a, b). Cognition-enhancing agents have been suggested as potential treatment medications for stimulant addiction (Sofuoglu, 2010), and some success 
with such agents has been observed in the treatment of cocaine (Dackis et al, 2005) and MA dependence (Shearer et al, 2009), but few studies have examined the direct effects of these potential pharmacotherapies on behavioral and neural measures of cognition in stimulant-abusing subjects. Modafinil appears to be safe and well-tolerated by MAdependent subjects (De La Garza et al, 2010; McGaugh et al, 2009), and some evidence suggests that it enhances the retention of $\mathrm{MA}$-dependent subjects in programs that involve contingency management and cognitive behavioral therapy (Heinzerling et al, 2010), but little is known about the effects of modafinil on cognitive function in MA-dependent individuals (see Kalechstein et al (2010) for an example of working-memory enhancement in this sample).

The mechanism of action of modafinil is complex, involving multiple neurotransmitter systems (see Minzenberg and Carter (2008) for review). This action includes inhibition of catecholamine transporters (Madras et al, 2006; Volkow et al, 2009; Zolkowska et al, 2009), thereby increasing extracellular dopamine and norepinephrine levels. Given the important role of dopamine for associative learning and reward processing (eg, Schultz et al, 1997), and the abnormalities in dopamine markers observed in MA subjects (Lee et al, 2009; Volkow et al, 2001a; Wilson et al, 1996), modafinil may be particularly well-suited as an agent that promotes enhanced learning via improved dopaminergic function in MA-dependent subjects.

Moreover, MA-dependent subjects exhibit abnormal structure and function of the anterior cingulate cortex (ACC) (London et al, 2005; Salo et al, 2009; Thompson et al, 2004), a cortical structure that is associated with reward processing and has a major projection to dopamine-rich ventral striatal areas (Haber and Knutson, 2010). In light of evidence that modafinil raises ACC activation from a hypoactive state in patient groups (eg, schizophrenia) commensurate with improving behavioral performance in some participants (Spence et al, 2005), ACC appears to be a likely cortical target for the potential cognition-enhancing benefits of modafinil in MA-dependent subjects.

The goal of this project was to determine the effects of modafinil on learning and neural function in MA-dependent individuals, as compared with healthy adults who do not abuse MA. In a randomized, within-subject, placebocontrolled, double-blind crossover design, participants performed an associative learning task while undergoing functional magnetic resonance imaging (fMRI). The associative learning task was selected on the basis of prior work showing that it elicits fMRI activation in neural circuitry important for learning (Ghahremani et al, 2010). Moreover, as preliminary findings indicated that performance on this task was worse in MA-dependent individuals than in healthy control subjects, and negatively correlated with dopamine D2/D3 receptor availability (Ghahremani et al, 2009), we expected modafinil to improve performance in MA-dependent individuals, given evidence that it can enhance dopaminergic function (Madras et al, 2006; Volkow et al, 2009). Brain activation related to performing the task was assessed, testing for modafinil's effects and the potential interactions of this effect with the participant group. Drawing from prior findings, we hypothesized that modafinil would enhance learning performance and boost ACC activation, particularly in MA-dependent individuals.

\section{RESEARCH PARTICIPANTS AND METHODS}

All procedures were approved by the UCLA Office for the Protection of Research Subjects. Participants were recruited using radio, Internet, and newspaper advertisements. Two groups were recruited: MA-dependent participants (MA group) who were not seeking treatment, and healthy control participants (control group) who exhibited no evidence of a current drug abuse problem. Participants gave written informed consent after receiving a detailed explanation of the study. Then they underwent eligibility screening using questionnaires, the Structured Clinical Interview for DSMIV (First et al, 1996), and medical examination. Self-reports on prior drug use were obtained using a questionnaire regarding substance-abuse history (Table 2). MA participants were required to meet the DSM-IV criteria for MA dependence, and to demonstrate recent MA use in a urine drug screen. Control participants were required to have no prior or current diagnosis of illicit drug abuse or dependence. Participants were excluded from the study for the following: current Axis I diagnosis (except MA dependence, nicotine dependence, marijuana abuse, or mood/anxiety disorder due to substance abuse in the MA group and nicotine dependence in both groups); use of psychotropic medications or substances, except some marijuana or alcohol (not meeting the DSM-IV criteria for dependence); central nervous system, cardiovascular, pulmonary, or systemic disease; HIV seropositivity, hepatic disease, or hematocrit $<32$; pregnancy; lack of fluency in English; and MRI ineligibility due to metal implants or claustrophobia. Subjects were asked whether they had visual problems. If they indicated difficulty with viewing at a close distance, corrective lenses were used to ensure adequate visual acuity when viewing the stimuli in the scanner.

MA participants who satisfied the eligibility requirements of the study were admitted to the UCLA General Clinical Research Center, and participated on a residential basis. Conditions for continued participation in the study included abstinence from MA and other illicit drugs of abuse, as verified by periodic drug screening: daily testing of urine in the first few days after admission to the inpatient unit until tests were negative, followed by testing every other day; screening was always done on days of MRI scanning before data collection. Control participants visited the laboratory only on test days, and were required to provide negative urine samples for illicit substances on days of testing. Participants received compensation in the form of cash, gift certificates, and vouchers.

Twenty-two MA-dependent and 28 healthy control adults participated in the study. Nine control and eight MA participants were excluded from analyses because of any of the following conditions that may have occurred during either of the two scanning sessions: behavioral performance on the learning task at the chance level, excessive head motion during scanning ( $>2 \mathrm{~mm}$ translational displacement, $>1.5$ degrees rotation), scanner artifacts in functional images (as determined through inspection of MELODIC components, see below), a urine test positive 
for a drug of abuse (other than nicotine), and malfunctioning of behavioral response hardware. In total, data from 35 participants (16 MA, 19 control) were included in the study (Tables 1 and 2).

Table I Demographic Characteristics of Research Participants

\begin{tabular}{|c|c|c|c|}
\hline & $\begin{array}{l}\text { Control } \\
(n=19)\end{array}$ & $\begin{array}{l}\text { MA-dependent } \\
\quad(n=16)\end{array}$ & $\begin{array}{c}\text { Group } \\
\text { comparison }\end{array}$ \\
\hline Age $\left(\right.$ years) ${ }^{\mathrm{a}}$ & $33.7(8.7)$ & $36.0(9.4)$ & $t(33)=-0.75$ \\
\hline Sex (males/females) & $9 / 10$ & $10 / 6$ & $\chi^{2}(1)=0.31$ \\
\hline Education (years) & | $4.2(1.5)$ & |2.8(1.9) & $t(33)=2.34 *$ \\
\hline $\mathrm{IQ}^{\mathrm{b}}$ & $112.2(9.5)$ & $96.5(10.7)$ & $t(3 \mathrm{I})=4.45 * * *$ \\
\hline Ethnicity & & & $\chi^{2}(9)=11.25$ \\
\hline Caucasian & 11 & 7 & \\
\hline Hispanic & 4 & 3 & \\
\hline African American & 2 & 0 & \\
\hline Asian & । & 2 & \\
\hline Native American & 0 & । & \\
\hline Other & । & 3 & \\
\hline
\end{tabular}

$* P<0.05$, **** $P<0.00$ I. These factors were used as covariates in analyses of behavioral data (see Results).

aData shown are means (standard deviation).

bMeasured using the Wechsler Test of Adult Reading (WTAR) (Wechsler, 200I).

Table 2 Self-Reported Drug Use

$\begin{array}{lcc}\text { Control } & \text { MA- } & \text { Group } \\ (n=19) & \begin{array}{c}\text { dependent } \\ (n=16)\end{array}\end{array}$

\begin{tabular}{|c|c|}
\hline \multicolumn{2}{|l|}{ Methamphetamine } \\
\hline $\begin{array}{l}\text { Days used in } 30 \text { days before } \\
\text { study }^{\mathrm{a}}\end{array}$ & $22.9(8.3)$ \\
\hline $\begin{array}{l}\text { Grams used in the week before } \\
\text { the study }\end{array}$ & $3.1(3.5)$ \\
\hline Years & $10.3(8.7)$ \\
\hline Days abstinent before testing ${ }^{\mathrm{b}}$ & | $1.5(1.4)$ \\
\hline
\end{tabular}

\begin{tabular}{|c|c|c|c|}
\hline \multicolumn{4}{|l|}{ Marijuana } \\
\hline $\begin{array}{l}\text { Days used in } 30 \text {-days } \\
\text { before studyc }\end{array}$ & $0.07(0.26)$ & $4.25(9.19)$ & $t(28)=1.695$ \\
\hline \multicolumn{4}{|l|}{ Alcohol } \\
\hline Drinks per day & $0.47(0.28)$ & $0.55(0.55)$ & $t(33)=-0.56$ \\
\hline \multicolumn{4}{|l|}{ Tobacco } \\
\hline No. of smokers/nonsmokers & $11 / 8$ & $14 / 2$ & $\chi^{2}(1)=2.42$ \\
\hline Cigarettes per day (smokers only) & $6.3(6.6)$ & $10.4(7.9)$ & $t(33)=-1.82^{c}$ \\
\hline Pack years & $5.4(8.8)$ & |3.| (16.3) & $t(23)=-0.98$ \\
\hline
\end{tabular}

${ }^{a}$ Data shown are means (standard deviation).

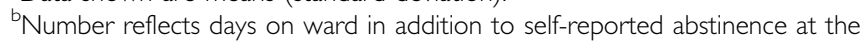
time of patient intake.

'Differences between groups did not reach statistical significance by Student's t-test $(P<0.1)$.
In a randomized double-blind placebo-controlled withinsubjects crossover design, participants received a single dose of modafinil $(200 \mathrm{mg})$ or a matching placebo pill $2 \mathrm{~h}$ before each scanning session. The dosage was determined on the basis of prior studies showing significant effects on cognition in patients with ADHD and schizophrenia with a single dose of this magnitude (Turner et al, 2004a; Turner et al, 2004b). The time between modafinil administration and testing was calculated on the basis of human pharmacokinetic data showing peak plasma levels at $2-4 \mathrm{~h}$ after a single dose (Robertson and Hellriegel, 2003).

Participants were randomly assigned to receive either modafinil or placebo first; randomization was determined by pharmacists who were not members of the research team. Nine control subjects and nine MA subjects received modafinil in the first session and placebo in the second session. Both scanning sessions were conducted at the same time of day $(\sim 1000$ hours for most subjects), and were separated by at least 2 days (mean $=2.56, \mathrm{SD}=0.89$ ) to allow washout of residual modafinil between sessions.

Given the effects of modafinil on wakefulness (see Repantis et al (2010) for a review), we administered the Piper Fatigue Scale (Piper et al, 1998) before participants entered the scanner, to assess the effects of the medication on sleepiness, energy levels, and mood. Education and IQ levels were compared across the groups as a means of assessing general differences in cognitive abilities. IQ was measured using the Wechsler Test of Adult Reading (Wechsler, 2001).

\section{Task and Design}

During scanning, participants performed a deterministic associative learning task with reversal components, which required selection of the correct response between two possible responses associated with a picture of an abstract visual pattern (Ghahremani et al, 2010) (Figure 1a). On each trial, participants were presented with a picture and were asked to decide whether it was associated with a left or right key response. The picture was presented for $1 \mathrm{~s}$, during which participants made their response. After this period, feedback appeared in the form of a colored square frame around the stimulus for $1 \mathrm{~s}$. A blue frame indicated a correct response, and a red frame indicated an incorrect response. If participants did not respond within the 1-s stimulus presentation period, the phrase 'no response recorded' appeared above the image. Participants received one point for a correct response and zero points for an incorrect response. A running total of points appeared beneath the stimulus during feedback presentation. Following presentation of feedback, a blank screen was displayed for a variable duration delay (inter-stimulus interval) of 0.5-16 s (sampled from an exponential distribution with a mean of $3 \mathrm{~s}$ ) before the next trial. Participants were encouraged to respond as quickly and as accurately as possible, and were told that their goal should be to accrue as many points possible.

After six repeated stimulus presentations, the stimulusresponse contingencies were reversed for some of the stimuli, such that the previously correct response became incorrect, requiring participants to adapt their responding flexibly to the newly correct alternative response. These reversals were intended for examination of the effect of 
a

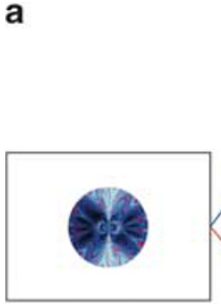

Stimulus: Abstract Picture (1s) Subject response (left or right key?)
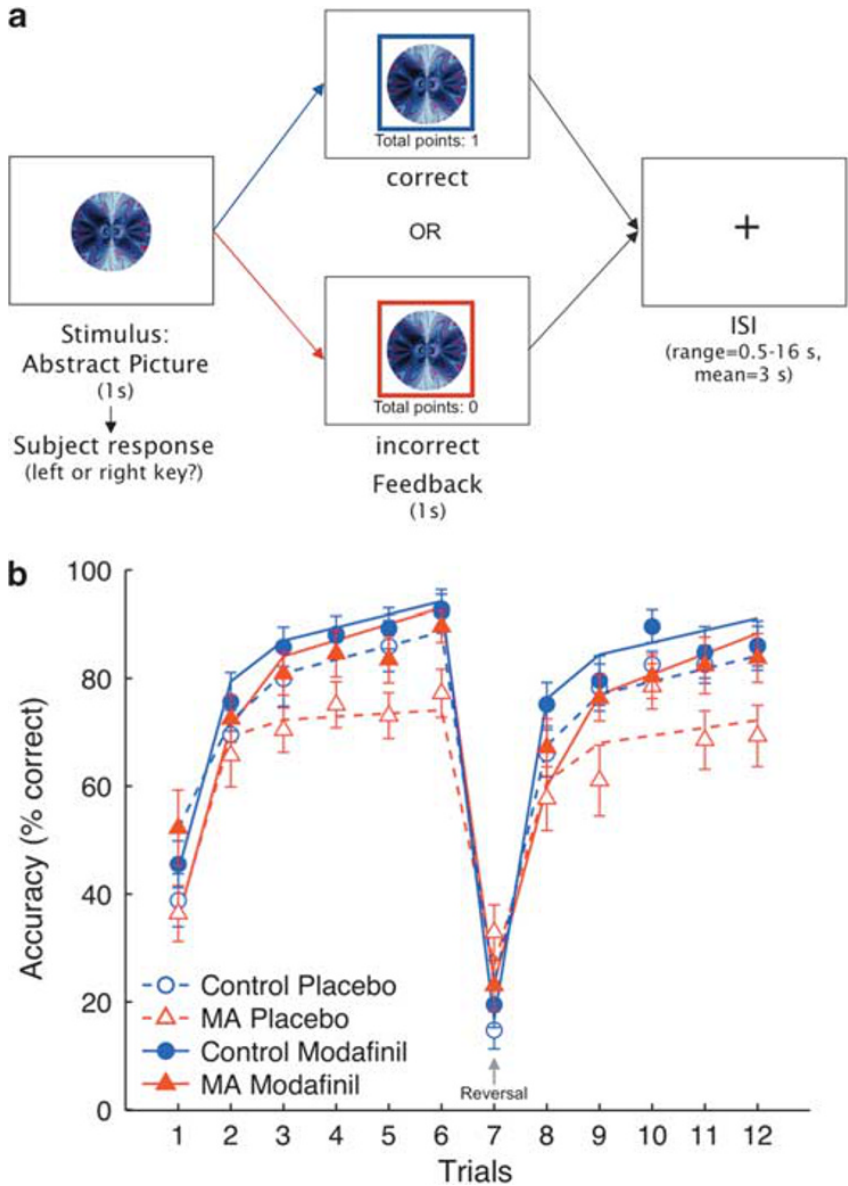

Figure I (a) Trial structure of reversal learning task. Participants were presented with an abstract image and were required to select the appropriate key (left or right key) within I s. If their response was correct, a blue frame appeared around the image, and they received I point (the display of "total points" was incremented by I). If their response was incorrect, a red frame appeared around the image and no points were gained or lost. After I s, a fixation cross appeared on the screen for an average inter-stimulus interval of $3 \mathrm{~s}$ (taken from an exponential distribution ranging from 0.5 to $16 \mathrm{~s}$ ). A total of 16 images were repeated 12 times in an interleaved, optimized sequence (see Research Participants and Methods). (b) Effect of modafinil on accuracy in the reversal learning task. Numbers on the $x$-axis indicate repeated trials for each of the 16 stimuli presented. The $y$-axis indicates percent correct responses at each of the 12 repeated trials. Triangles and circles represent mean values, and error bars each show one standard error of the mean. Plot lines reflect trajectories of the general linear mixed model (see methods). The within-subject learning trajectories were significantly related to group membership, treatment condition, trial repetitions, and the period (initial/late) within acquisition and reversal learning phases (group $\times$ med $\times$ repetition $\times$ period $(F(I, 77 I)=6.92$, $P=0.009$ ). The onset of the reversal stage in which stimulus-response contingencies changed is indicated by the arrow at the 7th stimulus repetition. MA-dependent group.

modafinil on cognitive flexibility. Participants were not explicitly informed about these response reversals.

To become familiarized with the task, participants performed the task outside the scanner in a behavioral testing session on a prior day. During each session in which the task was administered (familiarization session and the two sessions involving administration of a test compound), a different set of pictures (stimuli) was used, and the sets were counterbalanced across sessions.
Twenty-two abstract computer-generated images (ArtMatic Pro, U\&I Software LLC) were used as stimuli in the task. Three separate sets of stimuli were used for each administration of the task. The presentation and timing of all stimuli and response events were programmed using Matlab (Mathworks, Natick, MA) and the Psychtoolbox (http://www.psychtoolbox.org) on an Apple PowerBook G4 running Mac OSX (Apple Computers, Cupertino, CA). Visual stimuli were presented using MRI-compatible goggles (Resonance Technologies, Van Nuys, CA).

Among the 22 stimuli presented in the task, 16 were repeated 12 times (6 acquisition and 6 reversal repetitions), and the remaining 6 were presented six times during the acquisition phase only. The six stimuli that were not presented in the reversal stage were intended to thwart participants' expectations that all stimuli reversed; they were excluded from analyses to ensure a balanced number of trials between acquisition and reversal. Trials were organized in 12 'miniblocks' such that four stimuli were presented in a random order six times within each miniblock. Performance accuracy was determined for each stimulus repetition separately, calculating the proportion of correct trials of 16 and converting these proportions to percentages.

The sequence of trials and inter-stimulus intervals were determined using an in-house algorithm that used a MonteCarlo method to optimize the GLM design matrix for maximal statistical efficiency. Miniblocks were presented sequentially. Trials within a miniblock were pseudorandomized such that no stimulus was repeated in succession. Each stimulus reversed only once and was phased out of the experiment once the assigned repetitions were completed. Participants were only required to keep four stimuli in mind at any given point of time.

\section{Brain Imaging}

Imaging was performed using a 3-T Siemens AG (Erlangen, Germany) Allegra MRI scanner. We acquired 240 functional $\mathrm{T} 2{ }^{*}$-weighted echoplanar images (EPI) (slice thickness, $4 \mathrm{~mm} ; 34$ slices; repetition time (TR), $2 \mathrm{~s}$; echo time (TE), $30 \mathrm{~ms}$; flip angle, $90^{\circ}$; matrix, $64 \times 64$; field of view (FOV), $200 \mathrm{~mm}$ ). Two additional volumes were discarded at the beginning of each run to allow for $\mathrm{T} 1$ equilibrium effects. For registration purposes, a T2-weighted matchedbandwidth high-resolution anatomical scan (same slice prescription as EPI) and a magnetization-prepared rapidacquisition gradient echo (MPRAGE) high-resolution scan were acquired for each participant. The MPRAGE scan was conducted in a separate session on a Siemens Sonata 1.5-T scanner (slice thickness: $1 \mathrm{~mm}$; 160 slices, TR, $1900 \mathrm{~ms}$; TE, $4.38 \mathrm{~ms}$; flip angle, 15; FOV, $256 \mathrm{~mm}$ ). The orientation for matched-bandwidth and EPI scans was oblique axial so as to maximize full-brain coverage and to optimize the signals from ventral prefrontal regions.

\section{Data Analysis}

Behavioral data. The time courses of performance accuracy were determined by computing the proportion of correct trials for each stimulus repetition separately. The time courses of accuracy across trials (trajectories) were analyzed 
using a general linear mixed model (see Figure $1 \mathrm{~b}$ for the plot of model parameter estimates). The repeated measures structure of the data was accounted for by including a random factor for participants. The within-subject accuracy trajectories were modeled using three structural variables that described the learning phase (acquisition, reversal), and, within each phase, stimulus repetitions (1-6) and initial/late learning - different slopes for the initial (repetitions 1,2 ) and later learning phases (repetitions 3, 4, 5, 6).

The two experimental variables of interest were treatment (modafinil, placebo), included as a within-subject factor, and group (MA, control), modeled as a between-subject factor.

To account for effects potentially driven by differences in characteristics of the two groups, we included the demographic variables that differed significantly between groups at $P<0.05$ (ie, participants' education level and IQ) as timeinvariant covariates. All behavioral and demographic data were analyzed using Matlab (Mathworks, Natick, MA) and the R statistical package (http://www.r-project.org).

Imaging data. Analysis of fMRI data was performed using the FSL (4.1) toolbox from the Oxford Centre for fMRI of the Brain (http://www.fmrib.ox.ac.uk/fsl). The image time course for each participant was first realigned to compensate for small head movements (Jenkinson et al, 2002), and all non-brain matter was removed using FSL's brain extraction tool. Images were denoised for motion-related artifacts using MELODIC-independent components analysis within FSL. Motion-related components were identified manually using a set of heuristics (Tohka et al, 2008), and the data were then reconstructed after removing the motion-related components. Data were spatially smoothed using a 6-mm full-width-half-maximum Gaussian kernel. Registration was conducted by a three-step procedure, whereby EPI images were first registered to the matchedbandwidth high-resolution structural image, then to the MPRAGE structural image, and finally into standard Montreal Neurological Institute (MNI) space (MNI avg152 template), using 12-parameter affine transformations (Jenkinson and Smith, 2001). Registration from MPRAGE structural images to standard space was further refined using FNIRT nonlinear registration (Andersson et al, $2007 a, b)$. Statistical analyses at the single-subject level were performed in native space, with the statistical maps normalized to standard space before higher-level analysis.

Whole-brain statistical analysis was performed using a multi-stage approach to implement a mixed-effects model treating participants as a random effects variable. Statistical modeling was first performed separately for each imaging run. Regressors of interest were created by convolving a delta function representing trial-onset times with a canonical (double-gamma) hemodynamic response function. All trials in which participants successfully responded were included in a single regressor. To control for differences in performance across sessions, we added a parametric modulation covariate (Buchel et al, 1998) in which the modulator was trial-by-trial performance accuracy. Correct trials were weighted with 1 , and incorrect trials with -1; the covariate was demeaned for the regression model. Trials in which participants omitted a response were modeled as a single nuisance covariate, and motion parameters were included as covariates of no interest to account for variance associated with residual motion not captured by the MELODIC-denoising procedure. Only the initial regressor that included all trials was examined as the contrast of interest.

For all analyses, time-series statistical analysis was carried out using FILM (FMRIB's Improved Linear Model) with local autocorrelation correction (Woolrich et al, 2001) after high-pass temporal filtering (Gaussian-weighted LSF straight-line fitting, with sigma $=33 \mathrm{~s}$ ).

Contrast images for runs within each session were combined using fixed-effects analyses. To determine the effects of modafinil, pairwise, fixed-effects analyses comparing contrast images from the two test sessions were first conducted for each subject. These images were then submitted for group analyses using random-effects analyses.

For between-group analyses, the FMRIB Local Analysis of Mixed Effects (FLAME1) module in FSL was used (Beckmann et al, 2003; Woolrich et al, 2004), and a twosample $t$-test was performed at each voxel for each contrast of interest. $Z$ (Gaussianized T)-statistic images were thresholded using cluster-corrected statistics with a height threshold of $Z>1.96$ (unless otherwise noted), and a cluster probability threshold of $P<0.05$, whole-brain corrected using the theory of Gaussian Random Fields (Worsley et al, 1992). All group analyses were subjected to robust outlier deweighting (Woolrich, 2008). Anatomical locations of activations were identified using the Harvard-Oxford Probabilistic Atlas, which is included in the FSL software package, and the sectional brain atlas of Duvernoy and Bourgouin (1999).

\section{RESULTS}

\section{Characteristics of Research Participants: Demographics and Drug Use}

The demographics and drug-use data obtained for the two groups are presented in Tables 1 and 2. The MA and control groups did not differ significantly on age, sex distribution, or ethnicity. They did, however, show a difference in level of education and IQ as measured with the Wechsler Test of Adult Reading (Wechsler, 2001), with MA participants having completed fewer years and having lower IQ values, respectively. The groups did not differ significantly in marijuana use and the number of alcoholic drinks per day in the 30 days before testing. They also did not differ significantly in proportion of cigarette smokers, pack years (an indication of exposure to cigarette smoke), or reported number of cigarettes smoked per day.

\section{Task Performance}

Performance accuracy is shown in Figure $1 \mathrm{~b}$ along with results from the mixed-effects linear regression model. Modafinil improved task performance in the MA group, but did not affect performance in the control group. The analyses indicated significance of the structural parameters that collectively described the learning trajectories (learning phase $(\mathrm{F}(1,771)=8.38, \quad P=0.003)$, stimulus repetitions $\left.(\mathrm{F}(1,771)=172.27), \quad P=1.15 \times 10^{-35}\right), \quad$ and $\quad$ initial/late 
learning $\left(\mathrm{F}(1,771)=115.57, P=3.25 \times 10^{-25}\right)$, showing that all of them were necessary to describe the within-subject trajectories.

The learning trajectories were significantly related to both experimental variables: group (MA, control) and treatment (modafinil, placebo). There was an overall significant effect of treatment on performance accuracy $(\mathrm{F}(1,771)=5.97$, $P=0.015$ ) and the learning trajectories (learning phase $\times$ stimulus repetitions $\times$ initial/late learning, $F(1,771)=$ 22.73, $P=2.22 \times 10^{-6}$ ), indicating that the shape of the trajectory curve differed between the modafinil and placebo conditions. A significant group $\times$ treatment interaction effect on stimulus repetitions $\times$ initial/late learning $(\mathrm{F}(1,771)=6.92, \quad P=0.009)$ showed that the effect of modafinil on the rate of learning was different between groups, across both learning phases (acquisition/reversal). Specifically, modafinil led to a greater increase in the rate of learning in the MA group than in the control group.

To ensure that differences in education and IQ did not affect the results, we also evaluated whether these variables in the model would affect the model fit. Neither variables nor any of the interaction terms that included them contributed to the model (based on assessment of the Bayesian Information Criteria); therefore, we removed them from the model for better parsimony.

Modafinil reduced sensory aspects of fatigue, including sleepiness and energy levels. A repeated-measures ANOVA with group and treatment as factors showed a main effect of treatment on the sensory subscale of the Piper Fatigue Scale $(\mathrm{F}(1,33)=7.89, P=0.008$; modafinil: mean $=3.18$, $\mathrm{SD}=1.76$; placebo: mean $=3.81, \quad \mathrm{SD}=2.19$ ), with no significant effects of group or group by treatment interaction. As with the education variable, we included sensory aspects of fatigue as a covariate in our model of behavioral performance to determine whether behavioral performance was mediated by general arousal, but found that it did not affect the model fit.

\section{fMRI Results}

Task-related brain activation within groups. In the control group, comparison of all trials during the modafinil sessions $v s$ the placebo sessions revealed a large cluster of activation in bilateral ventral occipito-temporal cortex, lateral occipital cortex, and superior parietal regions (Supplementary Figure S1A and Supplementary Table S2), as well as a right lateralized activation in the pars triangularis region of the inferior frontal and middle frontal gyri. The MA group showed activations in the same regions as observed for the control group, and also in bilateral anterior insula, bilateral inferior frontal gyrus (including pars opercularis), orbitofrontal cortex, right middle temporal gyrus, hippocampus, and amygdala, caudate and midbrain (Supplementary Figure S1B and Supplementary Table S2). No regions showed greater activation in the placebo than in the modafinil condition in either group.

Comparisons within the modafinil condition between groups revealed greater activation in the MA group than in the control group in the anterior cingulate, left anterior insula/lateral orbitofrontal cortex, and the accumbens/ ventral striatal region (Supplementary Table S1). No

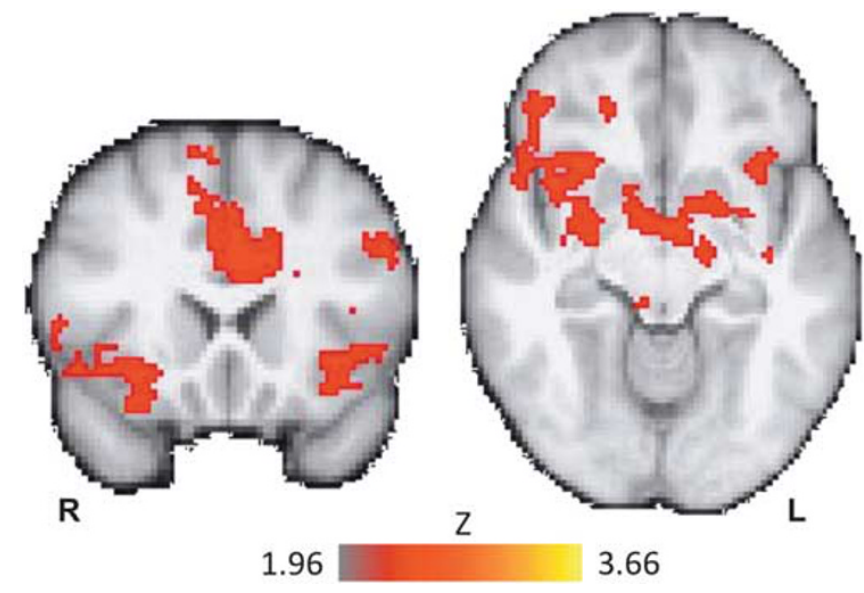

Figure 2 Task-related $\mathrm{fMRI}$ activity (all trials) corresponding to the interaction of treatment and group. The MA group showed a greater response to modafinil than did the control group in the anterior cingulate, bilateral anterior insula/ventrolateral prefrontal cortex, right inferior frontal gyrus, orbito-frontal cortex, and striatum (interaction: MA(modafinilplacebo) $>$ control(modafinil-placebo), see Table 3 for the full list of regions). Image shows $Z$-statistic map thresholded at $Z<1.96$ (cluster corrected, $P<0.05$ ) overlaid on a group-averaged high-resolution anatomical image. $M N I$ coordinates: coronal slice (left), $Y=20$, transverse slice (right), $Z=-\mid 0 . R=$ right, $L=$ left. Color bar indicates $Z$-statistic range.

significant differences were found between groups in the placebo condition.

Brain activation related to interaction of treatment and group. Given our observation of a group by treatment interaction in performance accuracy, we examined brain activation associated with this interaction. Brain regions that showed a greater effect of modafinil in the MA group than in the control group (contrast: MA (modafinilplacebo) $>$ control (modafinil-placebo)) included the anterior cingulate, bilateral anterior and middle insula, bilateral inferior frontal gyrus, putamen, and right amygdala (Figure 2, Table 3). No regions showed a greater treatment effect for the control group than the MA groups (control (modafinil-placebo) $>$ MA (modafinil-placebo $)$ ).

\section{DISCUSSION}

As expected from prior reports on cognitive performance in MA-dependent subjects, learning performance of the MA group was worse than that of the control group in the placebo condition. However, modafinil improved the performance of the MA group to levels equivalent to those of the control group. This improvement in performance of the MA group was commensurate with greater activation in the bilateral insula, inferior frontal gyrus, and ACC during task performance. These activations were not simply related to performance differences across sessions, as we controlled for correct/incorrect responses in our fMRI analyses.

The greater impact of modafinil on learning performance in the MA group than in healthy control subjects is consistent with previous observations that individuals who perform poorly on cognitive tasks, relative to those who perform well, show greater improvement after receiving modafinil (Finke et al, 2010; Kalechstein et al, 2010; Spence 
Table 3 Brain Regions of Significant Task-Related Activity (all trials) Showing the Interaction of Group (MA/control) and Treatment Condition (modafinil/placebo)

\begin{tabular}{|c|c|c|c|c|}
\hline Location & Z-stat & $\begin{array}{c}X \\
(\mathrm{~mm})\end{array}$ & $\begin{array}{c}Y \\
(\mathrm{~mm})\end{array}$ & $\begin{array}{c}Z \\
(\mathrm{~mm})\end{array}$ \\
\hline \multicolumn{5}{|l|}{ Right } \\
\hline $\begin{array}{l}\text { Inferior frontal gyrus, pars } \\
\text { opercularis/frontal operculum }\end{array}$ & 3.32 & 50 & 14 & 0 \\
\hline Inferior frontal gyrus, pars triangularis & 2.54 & 50 & 26 & -6 \\
\hline Superior frontal gyrus & 3.32 & 18 & 44 & 30 \\
\hline \multirow[t]{2}{*}{ Middle frontal gyrus } & 2.76 & 40 & 52 & 8 \\
\hline & 2.71 & 26 & 58 & 16 \\
\hline Precuneus & 2.42 & 16 & -68 & 30 \\
\hline Lateral occipital cortex & 2.63 & 40 & -58 & 46 \\
\hline Amygdala & 2.05 & 26 & 0 & -14 \\
\hline \multicolumn{5}{|l|}{ Left } \\
\hline $\begin{array}{l}\text { Inferior frontal gyrus, pars } \\
\text { opercularis/precentral gyrus }\end{array}$ & 2.51 & -48 & 18 & 32 \\
\hline Precentral sulcus & 2.85 & -54 & -4 & 16 \\
\hline \multicolumn{5}{|l|}{ Bilateral } \\
\hline Anterior cingulate cortex & 3.68 & 2 & 26 & 30 \\
\hline \multirow[t]{2}{*}{ Insular cortex } & 2.54 & 32 & 20 & -8 \\
\hline & 2.63 & -36 & 16 & -8 \\
\hline \multirow[t]{2}{*}{ Putamen } & 2.93 & 30 & 2 & -6 \\
\hline & 2.43 & -18 & 10 & -4 \\
\hline
\end{tabular}

Abbreviation: MA, methamphetamine-dependent group.

$Z$-statistics and $X, Y$, and $Z M N I$ coordinates $(\mathrm{mm})$ are from the location of peak voxel activation within each cluster. $Z$-statistic maps were whole-brain clustercorrected at $Z=1.96, P<0.05$. Interaction direction: MA (modafinil-

placebo) > control (modafinil-placebo); no regions showed significant clusters in the opposite direction (control (modafinil-placebo) > MA (modafinil-placebo)).

et al, 2005). Indeed, in the present study, a post hoc analysis of low- and high-performing control participants (determined by a median split of performance accuracy during placebo conditions) showed a bigger effect of modafinil on performance for low- than for high-performing participants (difference in accuracy between modafinil and placebo conditions: low performers, $8 \% v s$ high performers, $0.7 \%$; $t(16)=2.68, P=0.02)$. Therefore, the lack of modafinil effect we observed in the control group as a whole was likely driven by the high performance of subjects who were already performing at near-ceiling levels with minimal room for improvement.

Our observation of greater activation of the ACC under modafinil than placebo conditions in the MA group has particular therapeutic relevance, as the ACC has been shown to be hypoactive in MA subjects (as compared with activity in control subjects) during tests of cognitive control (Salo et al, 2009) and vigilance (London et al, 2005). The boost in ACC activation observed with modafinil likely reflects greater engagement of cognitive processes important for learning, especially as ACC activation has been previously shown to correlate positively with learning performance on this task in control subjects (Ghahremani et al, 2010).
Similar correlations have been observed in schizophrenic patients on modafinil between working-memory performance and ACC activation (Spence et al, 2005). The fact that we did not observe a modafinil effect on ACC activation in control subjects, coinciding with a null behavioral effect of the treatment in this group, suggests that modafinil influenced an improvement in MA behavioral performance by facilitating activation in this region.

In addition to the ACC, the anterior insula/ventrolateral prefrontal cortex also showed greater bilateral activation during modafinil than placebo conditions in the MA group, a difference that was not found in the control group. As activation of the anterior insula and ventrolateral prefrontal cortex is commonly observed in studies of cognitive control (eg, Cools et al, 2002; Ghahremani et al, 2010), and as MA subjects show neurocognitive deficits associated with this region (London et al, 2005; Tabibnia et al, in press), it is possible that the anterior insula/ventrolateral prefrontal activation is related to enhanced cognitive control associated with improved task performance.

The insula has been implicated in a range of psychological processes that have been characterized as involving interoceptive awareness (Craig, 2009), with meta-analyses suggesting that it integrates multiple functions to provide a coherent world experience (Kurth et al, 2010). Given its involvement in awareness of subjective states, along with reports of damage to this region reducing craving in cigarette smokers (Naqvi et al, 2007) and its activation associated with drug craving (Bonson et al, 2002; Brody et al, 2002; Filbey et al, 2009), the insula has been highlighted as having an important role in substance abuse (Garavan, 2010; Naqvi and Bechara, 2009). Although the specific mechanism by which the insula influences substance-abuse behavior is not yet clear, further empirical investigations are warranted to determine the therapeutic relevance of pharmacological manipulations of insula activation for improving treatment outcomes in MA dependence.

Despite evidence for modafinil operating on multiple neurochemical systems in the brain, including those involving serotonin, glutamate, gamma aminobutyric acid, hypocretin/orexin, and histamine, its effects on catecholamines have been proposed to primarily underlie cognitive enhancement (Minzenberg and Carter, 2008). Modafinil has been shown to elevate extracellular levels of dopamine in humans by blocking the dopamine transporter (DAT) (Volkow et al, 2009), and dopamine regulation in both the anterior cingulate and insular cortices by DAT has been demonstrated in humans (Ciliax et al, 1999). Both the ACC and the dopamine-rich striatal areas to which it projects comprise major neural circuitry, which supports the associative learning ability examined in our study (Haber and Knutson, 2010). Given that MA subjects show reduced striatal DAT availability related to poor learning and memory (McCann et al, 2008; Volkow et al, 2001b), modafinil likely operates on the DAT in these regions, enhancing dopamine transmission to facilitate learning.

The reason for the mismatch between modafinil-induced increases in brain activation in control participants without improvement in performance is not clear. One explanation is that the activation was driven by participants with low performance who showed a behavioral enhancement with 
modafinil. Unfortunately, separately analyzing fMRI data from low- and high-performing participants was not feasible because of the small sample sizes. However, discrepancies between behavioral performance and brain activation when examining modafinil are not unique to this study. A prior fMRI study in which modafinil $(100 \mathrm{mg})$ was given daily for 7 days showed no effect of the medication on performance in a test of attentional control, but reduced ACC activation during task performance (Rasetti et al, 2010), also showing a mismatch between behavioral and fMRI effects of modafinil. Moreover, it is important to note that the mixed results found for the cognition-enhancing effects of modafinil on behavior in healthy participants often depend on the particular behavioral task, measures employed, and dosing regimens (Repantis et al, 2010). It is possible that the specific learning paradigm or the particular dosing procedure (single-dose of $200 \mathrm{mg}$ ) used in this study was not sensitive enough to capture measurable cognition-enhancing effects on performance (at least, in the participants with high performance), which may have been reflected in brain activation differences.

Although we found improved learning performance with modafinil in the MA group in functional circuits important for learning, we cannot rule out the possibility that modafinil may have had a more general effect of increasing motivation and vigilance among participants, as has been shown previously in rodents (Young and Geyer, 2010) and humans (Baranski et al, 2004).

Our study used a learning task to assess prefrontal function because of the importance of learning for engagement in behavioral therapies, and evidence that modafinil can enhance learning and memory (eg, Turner et al, 2003). Further neuromaging studies of modafinil are required to examine its effects on the neural substrates underlying other important cognitive functions, such as response inhibition and decision making, that are often compromised in substance abuse.

With continued interest in modafinil as a potential pharmacotherapy for stimulant abuse (Karila et al, 2010), our results indicate that improvements in cognitive function elicited by the medication may augment traditional behavioral therapies and increase their efficacy. Modafinil or other cognition-enhancing medications may therefore be useful adjuncts to behavioral treatments for stimulant dependence.

\section{ACKNOWLEDGEMENTS}

This work was supported by NIH grants P20 DA022539, R01 DA020726 (EDL), the Consortium for Neuropsychiatric Phenomics (NIH Roadmap for Medical Research Grant UL1 DE019580, RL1 DA024853 (EDL)), and M01 RR00865 (UCLA GCRC). Additional funding was provided by endowments from the Thomas $\mathrm{P}$ and Katherine $\mathrm{K}$ Pike Chair in Addiction Studies, and the Marjorie M Greene Trust.

\section{DISCLOSURE}

Research support for projects other than the one reported here was supplied to Dr Edythe London under UCLA contract (number 20063287) with Philip Morris USA. There was no involvement of Philip Morris USA in this project. None of the other authors have any conflict of interest or financial disclosures to report.

\section{REFERENCES}

Andersson J, Jenkinson M, Smith S (2007a). Non-linear optimisation. FMRIB Technical Report.

Andersson J, Jenkinson M, Smith S (2007b). Non-linear registration, aka Spatial normalisation. FMRIB Technical Report.

Baranski JV, Pigeau R, Dinich P, Jacobs I (2004). Effects of modafinil on cognitive and meta-cognitive performance. Hum Psychopharmacol 19: 323-332.

Beckmann CF, Jenkinson M, Smith SM (2003). General multilevel linear modeling for group analysis in FMRI. Neuroimage 20: 1052-1063.

Bonson KR, Grant SJ, Contoreggi CS, Links JM, Metcalfe J, Weyl HL et al (2002). Neural systems and cue-induced cocaine craving. Neuropsychopharmacology 26: 376-386.

Brody AL, Mandelkern MA, London ED, Childress AR, Lee GS, Bota RG et al (2002). Brain metabolic changes during cigarette craving. Arch Gen Psychiatry 59: 1162-1172.

Buchel C, Holmes AP, Rees G, Friston KJ (1998). Characterizing stimulus-response functions using nonlinear regressors in parametric fMRI experiments. Neuroimage 8: 140-148.

Ciliax BJ, Drash GW, Staley JK, Haber S, Mobley CJ, Miller GW et al (1999). Immunocytochemical localization of the dopamine transporter in human brain. J Comp Neurol 409: 38-56.

Cools R, Clark L, Owen AM, Robbins TW (2002). Defining the neural mechanisms of probabilistic reversal learning using event-related functional magnetic resonance imaging. J Neurosci 22: $4563-4567$.

Craig AD (2009). How do you feel-now? The anterior insula and human awareness. Nat Rev Neurosci 10: 59-70.

Dackis CA, Kampman KM, Lynch KG, Pettinati HM, O'Brien CP (2005). A double-blind, placebo-controlled trial of modafinil for cocaine dependence. Neuropsychopharmacology 30: 205-211.

De La Garza II R, Zorick T, London ED, Newton TF (2010). Evaluation of modafinil effects on cardiovascular, subjective, and reinforcing effects of methamphetamine in methamphetamine-dependent volunteers. Drug Alcohol Depend 106: 173-180.

Duvernoy HM, Bourgouin P (1999). The Human Brain: Surface, Three-Dimensional Sectional Anatomy with MRI, and Blood Supply, 2nd completely rev. and enl. edn. Springer: Wien, New York. p 491.

Filbey FM, Schacht JP, Myers US, Chavez RS, Hutchison KE (2009). Marijuana craving in the brain. Proc Natl Acad Sci USA 106: 13016-13021.

Finke K, Dodds CM, Bublak P, Regenthal R, Baumann F, Manly T et al (2010). Effects of modafinil and methylphenidate on visual attention capacity: a TVA-based study. Psychopharmacology (Berl) 210: 317-329.

First MB, Spitzer RL, Gibbon M, Williams J (1996). Structured Clinical Interview for DSM-IV Axis I Disorders- Patient Edition (SCID-IP, Version 2.0). Biometrics Research Department, New York State Psychiatric Institute: New York, NY.

Garavan H (2010). Insula and drug cravings. Brain Struct Funct 214: $593-601$.

Ghahremani DG, Lee B, Tabibnia G, Monterosso J, Poldrack RA, London ED (2009). Impulsive behavior and dopamine D2/3 receptor availability in healthy and MA-dependent individuals. In: 2009 Neuroscience Meeting Planner Society for Neuroscience (online).

Ghahremani DG, Monterosso J, Jentsch JD, Bilder RM, Poldrack RA (2010). Neural components underlying behavioral flexibility in human reversal learning. Cereb Cortex 20: 1843-1852. 
Gonzales R, Mooney L, Rawson RA (2010). The methamphetamine problem in the United States. Annu Rev Public Health 31: 385-398.

Greely H, Sahakian B, Harris J, Kessler RC, Gazzaniga M, Campbell $\mathrm{P}$ et al (2008). Towards responsible use of cognitive-enhancing drugs by the healthy. Nature 456: 702-705.

Haber SN, Knutson B (2010). The reward circuit: linking primate anatomy and human imaging. Neuropsychopharmacology 35: 4-26.

Heinzerling KG, Swanson AN, Kim S, Cederblom L, Moe A, Ling W et al (2010). Randomized, double-blind, placebo-controlled trial of modafinil for the treatment of methamphetamine dependence. Drug Alcohol Depend 109: 20-29.

Jenkinson M, Bannister P, Brady M, Smith S (2002). Improved optimization for the robust and accurate linear registration and motion correction of brain images. Neuroimage 17: 825-841.

Jenkinson M, Smith S (2001). A global optimisation method for robust affine registration of brain images. Med Image Anal 5: 143-156.

Kalechstein AD, De La Garza II R, Newton TF (2010). Modafinil administration improves working memory in methamphetamine-dependent individuals who demonstrate baseline impairment. Am J Addict 19: 340-344.

Karila L, Weinstein A, Aubin HJ, Benyamina A, Reynaud M, Batki SL (2010). Pharmacological approaches to methamphetamine dependence: a focused review. Br J Clin Pharmacol 69: 578-592.

Kurth F, Zilles K, Fox PT, Laird AR, Eickhoff SB (2010). A link between the systems: functional differentiation and integration within the human insula revealed by meta-analysis. Brain Struct Funct 214: 519-534.

Lee B, London ED, Poldrack RA, Farahi J, Nacca A, Monterosso JR et al (2009). Striatal dopamine $\mathrm{d} 2 / \mathrm{d} 3$ receptor availability is reduced in methamphetamine dependence and is linked to impulsivity. J Neurosci 29: 14734-14740.

Lee NK, Rawson RA (2008). A systematic review of cognitive and behavioural therapies for methamphetamine dependence. Drug Alcohol Rev 27: 309-317.

London ED, Berman SM, Voytek B, Simon SL, Mandelkern MA, Monterosso J et al (2005). Cerebral metabolic dysfunction and impaired vigilance in recently abstinent methamphetamine abusers. Biol Psychiatry 58: 770-778.

Madras BK, Xie Z, Lin Z, Jassen A, Panas H, Lynch L et al (2006). Modafinil occupies dopamine and norepinephrine transporters in vivo and modulates the transporters and trace amine activity in vitro. J Pharmacol Exp Ther 319: 561-569.

McCann UD, Kuwabara H, Kumar A, Palermo M, Abbey R, Brasic J et al (2008). Persistent cognitive and dopamine transporter deficits in abstinent methamphetamine users. Synapse 62: 91-100

McGaugh J, Mancino MJ, Feldman Z, Chopra MP, Gentry WB, Cargile C et al (2009). Open-label pilot study of modafinil for methamphetamine dependence. J Clin Psychopharmacol 29: 488-491.

Minzenberg MJ, Carter CS (2008). Modafinil: a review of neurochemical actions and effects on cognition. Neuropsychopharmacology 33: 1477-1502.

Monterosso JR, Aron AR, Cordova X, Xu J, London ED (2005). Deficits in response inhibition associated with chronic methamphetamine abuse. Drug Alcohol Depend 79: 273-277.

Naqvi NH, Bechara A (2009). The hidden island of addiction: the insula. Trends Neurosci 32: 56-67.

Naqvi NH, Rudrauf D, Damasio H, Bechara A (2007). Damage to the insula disrupts addiction to cigarette smoking. Science 315: 531-534.

Piper BF, Dibble SL, Dodd MJ, Weiss MC, Slaughter RE, Paul SM (1998). The revised Piper Fatigue Scale: psychometric evaluation in women with breast cancer. Oncol Nurs Forum 25: 677-684.
Pluddemann A, Flisher AJ, McKetin R, Parry C, Lombard C (2010). Methamphetamine use, aggressive behavior and other mental health issues among high-school students in Cape Town, South Africa. Drug Alcohol Depend 109: 14-19.

Randall DC, Viswanath A, Bharania P, Elsabagh SM, Hartley DE, Shneerson JM et al (2005). Does modafinil enhance cognitive performance in young volunteers who are not sleep-deprived? J Clin Psychopharmacol 25: 175-179.

Rasetti R, Mattay VS, Stankevich B, Skjei K, Blasi G, Sambataro F et al (2010). Modulatory effects of modafinil on neural circuits regulating emotion and cognition. Neuropsychopharmacology 35: 2101-2109.

Rawson RA, Condon TP (2007). Why do we need an addiction supplement focused on methamphetamine? Addiction 102(Suppl 1): $1-4$.

Repantis D, Schlattmann P, Laisney O, Heuser I (2010). Modafinil and methylphenidate for neuroenhancement in healthy individuals: a systematic review. Pharmacol Res 62: 187-206.

Robertson Jr P, Hellriegel ET (2003). Clinical pharmacokinetic profile of modafinil. Clin Pharmacokinet 42: 123-137.

Sahakian B, Morein-Zamir S (2007). Professor's little helper. Nature 450: 1157-1159.

Salo R, Nordahl TE, Moore C, Waters C, Natsuaki Y, Galloway GP et al (2005). A dissociation in attentional control: evidence from methamphetamine dependence. Biol Psychiatry 57: 310-313.

Salo R, Ursu S, Buonocore MH, Leamon MH, Carter C (2009). Impaired prefrontal cortical function and disrupted adaptive cognitive control in methamphetamine abusers: a functional magnetic resonance imaging study. Biol Psychiatry 65: 706-709.

Schultz W, Dayan P, Montague PR (1997). A neural substrate of prediction and reward. Science 275: 1593-1599.

Scott JC, Woods SP, Matt GE, Meyer RA, Heaton RK, Atkinson JH et al (2007). Neurocognitive effects of methamphetamine: a critical review and meta-analysis. Neuropsychol Rev 17: 275-297.

Shearer J, Darke S, Rodgers C, Slade T, van Beek I, Lewis J et al (2009). A double-blind, placebo-controlled trial of modafinil (200 mg/day) for methamphetamine dependence. Addiction 104: 224-233.

Simon SL, Dean AC, Cordova X, Monterosso JR, London ED (2010). Methamphetamine dependence and neuropsychological functioning: evaluating change during early abstinence. J Stud Alcohol Drugs 71: 335-344.

Sofuoglu M (2010). Cognitive enhancement as a pharmacotherapy target for stimulant addiction. Addiction 105: 38-48.

Spence SA, Green RD, Wilkinson ID, Hunter MD (2005). Modafinil modulates anterior cingulate function in chronic schizophrenia. Br J Psychiatry 187: 55-61.

Stix G (2009). Turbocharging the brain. Sci Am 301: 46-49, 52-45.

Tabibnia G, Monterosso JR, Baicy JK, Chakrapani S, Lee B, Aron $\mathrm{AR}$ et al (in press). Different forms of self-control share a neurocognitive substrate. J Neurosci.

Thompson PM, Hayashi KM, Simon SL, Geaga JA, Hong MS, Sui Y et al (2004). Structural abnormalities in the brains of human subjects who use methamphetamine. J Neurosci 24: 6028-6036.

Tohka J, Foerde K, Aron AR, Tom SM, Toga AW, Poldrack RA (2008). Automatic independent component labeling for artifact removal in fMRI. Neuroimage 39: 1227-1245.

Turner DC, Clark L, Dowson J, Robbins TW, Sahakian BJ (2004a). Modafinil improves cognition and response inhibition in adult attention-deficit/hyperactivity disorder. Biol Psychiatry 55: 1031-1040.

Turner DC, Clark L, Pomarol-Clotet E, McKenna P, Robbins TW, Sahakian BJ (2004b). Modafinil improves cognition and attentional set shifting in patients with chronic schizophrenia. Neuropsychopharmacology 29: 1363-1373.

Turner DC, Robbins TW, Clark L, Aron AR, Dowson J, Sahakian BJ (2003). Cognitive enhancing effects of modafinil in healthy volunteers. Psychopharmacology (Berl) 165: 260-269. 
United Nations Office on Drugs and Crime (2009). World Drug Report 2009. UN Office on Drugs and Crime: Vienna.

Vocci FJ (2008). Cognitive remediation in the treatment of stimulant abuse disorders: a research agenda. Exp Clin Psychopharmacol 16: 484-497.

Volkow ND, Chang L, Wang GJ, Fowler JS, Ding YS, Sedler M et al (2001a). Low level of brain dopamine D2 receptors in methamphetamine abusers: association with metabolism in the orbitofrontal cortex. Am J Psychiatry 158: 2015-2021.

Volkow ND, Chang L, Wang GJ, Fowler JS, Leonido-Yee M, Franceschi D et al (2001b). Association of dopamine transporter reduction with psychomotor impairment in methamphetamine abusers. Am J Psychiatry 158: 377-382.

Volkow ND, Fowler JS, Logan J, Alexoff D, Zhu W, Telang F et al (2009). Effects of modafinil on dopamine and dopamine transporters in the male human brain: clinical implications. JAMA 301: 1148-1154.

Wechsberg WM, Jones HE, Zule WA, Myers BJ, Browne FA, Kaufman MR et al (2010). Methamphetamine ('tik') use and its association with condom use among out-of-school females in Cape Town, South Africa. Am J Drug Alcohol Abuse 36: 208-213.

Wechsler D (2001). Wechsler Test of Adult Reading (WTAR). The Psychological Corporation: San Antonio.
Wesensten NJ, Belenky G, Kautz MA, Thorne DR, Reichardt RM, Balkin TJ (2002). Maintaining alertness and performance during sleep deprivation: modafinil versus caffeine. Psychopharmacology (Berl) 159: 238-247.

Wilson JM, Kalasinsky KS, Levey AI, Bergeron C, Reiber G, Anthony RM et al (1996). Striatal dopamine nerve terminal markers in human, chronic methamphetamine users. Nat Med 2: 699-703.

Woolrich M (2008). Robust group analysis using outlier inference. Neuroimage 41: 286-301.

Woolrich MW, Behrens TE, Beckmann CF, Jenkinson M, Smith SM (2004). Multilevel linear modelling for FMRI group analysis using Bayesian inference. Neuroimage 21: 1732-1747.

Woolrich MW, Ripley BD, Brady M, Smith SM (2001). Temporal autocorrelation in univariate linear modeling of FMRI data. Neuroimage 14: 1370-1386.

Worsley KJ, Evans AC, Marrett S, Neelin P (1992). A threedimensional statistical analysis for CBF activation studies in human brain. J Cereb Blood Flow Metab 12: 900-918.

Young JW, Geyer MA (2010). Action of modafinil-increased motivation via the dopamine transporter inhibition and D1 receptors? Biol Psychiatry 67: 784-787.

Zolkowska D, Jain R, Rothman RB, Partilla JS, Roth BL, Setola V et al (2009). Evidence for the involvement of dopamine transporters in behavioral stimulant effects of modafinil. J Pharmacol Exp Ther 329: 738-746.

Supplementary Information accompanies the paper on the Neuropsychopharmacology website (http://www.nature.com/npp) 